\title{
Etude épidémiologique de la prise en charge des patients sous AVK au cabinet dentaire en Ille-et-Vilaine
}

\author{
Clipet F, Massot M, Garnier J, Kholer B, De Mello G \\ Service de Chirurgie Buccale, CHU, Rennes, France \\ Faculté d'Odontologie, Université de Rennes 1, France \\ fabrice.clipet@wanadoo.fr
}

En odontologie, la prise en charge des patients sous traitement anticoagulant, et en particulier sous antivitamines $\mathrm{K}(\mathrm{AVK})$, est en pleine évolution. Actuellement, environ 1,5\% de la population française est traité par AVK, soit environ 600000 personnes. La majorité de ces patients est représentée par des personnes âgées, très souvent polymédiquées et donc fragiles (Persac 2007).

Depuis 2006, la Société Française de Cardiologie (SFC) et la Société Francophone de Médecine buccale et de Chirurgie buccale (SFMBCB 2006) recommandent la poursuite du traitement pour un acte de chirurgie buccale. En effet, le traitement anticoagulant a pour but de diminuer le risque thromboembolique ; l'arrêter peut avoir des conséquences létales pour le patient (Della Valle 2003). La réalisation d'un relais aux héparines augmente le risque thromboembolique, ne met pas à l'abri d'hémorragies post-opératoires (Dunn 2003) et perturbe le traitement hypocoagulant qui sera par la suite difficile à ajuster et à stabiliser.

Afin d'évaluer les pratiques actuelles de prise en charge des patients sous AVK, nous avons effectué une étude épidémiologique sur la pratique des chirurgiens dentistes d'Ille-et-Vilaine à l'aide d'un questionnaire. Le recueil des données a été réalisé en 2007. Un courrier informatif décrivant l'étude, un questionnaire et une enveloppe timbrée avec l'adresse pré-inscrite a été envoyé par courrier aux 730 chirurgiens dentistes d'Ille-et-Vilaine durant le mois de mai 2007. Les questions reprennent les différents protocoles opératoires mis en place lors de la réalisation d'un acte chirurgical invasif au cabinet dentaire.

Sur 730 questionnaires envoyés, 312 ont été retournés, soit 42,74\% de réponses. Parmi ces résultats, 12 sont inexploitables. Sur les 300 questionnaires exploitables, 170 praticiens $(56,67 \%)$ prennent en charge les patients sous AVK lors d'actes sanglants : 56 hommes $(38,62 \%)$ et 114 femmes $(73,55 \%)$. Sur ces 170 praticiens, $27(15,88 \%)$ choisissent de ne pas arrêter le traitement anticoagulant dans la plupart des cas, sinon ils demandent un relais ou l"arrêt du traitement AVK. $28(16,47 \%)$ choisissent strictement de ne pas arrêter le traitement AVK. Parmi les 28 praticiens respectant le protocole de la SFMBCB, 17 ont indiqué la valeur limite de l'INR pour laquelle ils réalisent un acte sanglant : les valeurs se situent entre 2,5 et 4,5 avec un INR moyen de 3 .

Notre étude a essayé de dresser un premier état des lieux de la prise en charge de ces patients en Ille-et-Vilaine et de rendre compte des pratiques. La presque totalité des chirurgiens dentistes de ce département a reçu le questionnaire et le grand nombre de réponses $(42,74 \%$ de réponses) donne un bon échantillon de la population de praticiens exerçant dans ce département. Les recommandations de la SFMBCB et de la SFC sont adoptées par une minorité de praticiens. Comme d'anciennes habitudes perdurent, il semble important que les différents acteurs de notre profession prennent une part active à la diffusion et à l'enseignement de ces recommandations. 